\title{
Effect of Probucol, Pantethine and Their Combinations on Serum Lipoprotein Metabolism and on the Incidence of Atheromatous Lesions in the Rabbit
}

\author{
Katsuhiko TAWARA, Masanao ISHIHARA, Hidemasa OGAWA \\ and Munehiro TOMIKAWA \\ Research Institute, Daiichi Seiyaku Co., Ltd., 16-13, Kitakasai 1-chome, \\ Edogawa-ku, Tokyo 134, Japan
}

Accepted March 6, 1986

\begin{abstract}
Effect of probucol, pantethine and their combinations on serum lipoprotein metabolism and on the incidence of atheromatous lesions in aorta and coronary artery was studied in cholesterol-fed rabbits. Probucol treatment $(0.5 \%$ in diet) resulted in reducing HDL cholesterol and serum apo $\mathrm{A}-\mathrm{I}$ levels significantly. while pantethine treatment $(0.25 \%-0.75 \%$ in diet) tended to increase HDL cholesterol and serum apo A-I levels. Combined treatment with these two drugs showed a significant prevention in the reduction of HDL cholesterol and serum apo A-I levels by probucol alone. Probucol or pantethine treatment reduced effectively (V) LDL cholesterol and serum apo B levels, and these effects were accelerated additively when the two drugs were given concurrently. Atheromatous lesions in aorta and coronary artery in cholesterol-fed rabbits were prevented by the treatment with probucol $(0.5 \%$ in diet) or pantethine $(0.75 \%$ in diet) for 24 weeks. The combined treatment with these two drugs showed more marked prevention than either drug alone. From these findings, it is concluded that the combined treatment of probucol with pantethine is effective for improvement of serum lipoprotein disorders and for prevention of the incidence of atheromatous lesions in aorta and coronary artery in cholesterol-fed rabbits.
\end{abstract}

Elevated cholesterol level, specifically high level of low density lipoprotein (LDL.) cholesterol in the blood stream, is believed to be one of the major causes of developing atherosclerotic disease, especially of the coronary artery (1-4). On the other hand, the inverse relationship between plasma levels of high density lipoprotein (HDL) and risk of coronary artery disease have been demonstrated in numerous studies (5-9).

Probucol treatment induces a marked reduction in total cholesterol and LDL cholesterol levels (10-15) concomitant with a significant reduction in HDL cholesterol and apo $\mathrm{A}-\mathrm{I}$ levels in serum $(16,17)$.

If the reduction of HDL cholesterol and serum apo $A-1$ levels by probucol could be prevented by the combination with other agents such as HDL cholesterol elevating agents, the hypocholesterolemic action of probucol might be more useful for the prevention of atherosclerotic lesion.

From this point of view, combined treatment with probucol and pantethine on serum lipids, lipoproteins and apolipoproteins were studied in cholesterol-fed rabbits in the present study, because pantethine elevated effectively HDL cholesterol and apo A-1 levels in serum as reported previously (18). Combined treatment with both agents on the incidence of atheromatous lesions in the aorta and coronary artery was also examined.

\section{Materials and Methods}

Treatment of animals: Male Japanese albino rabbits weighing $2.0-2.5 \mathrm{~kg}$ (Kanamaru Laboratory Animal Center, Tokyo) were divided at random into 9 groups of 8 animals 
each after a 2-week acclimation period with normal standard laboratory chow (Oriental RC-4, Oriental Co., Tokyo). The 9 groups were treated for 9 weeks, respectively, with 1) normal standard laboratory chow (group 1). 2) $0.5 \%$ cholesterol diet (group II), 3) $0.5 \%$ cholesterol diet $+0.25 \%$ pantethine (group III), 4) $0.5 \%$ cholesterol diet $+0.5 \%$ pantethine (group IV), 5) $0.5 \%$ cholesterol diet $+0.75 \%$ pantethine (group $V$ ), 6) $0.5 \%$ cholesterol diet $+0.5 \%$ probucol (group $\mathrm{VI}$ ). 7) $0.5 \%$ cholesterol diet $+0.5 \%$ probucol $+0.25 \%$ pantethine (group VII), 8) $0.5 \%$ cholesterol diet $+0.5 \%$ probucol $+0.5 \%$ pantethine (group VIII) and 9) $0.5 \%$ cholesterol diet $+0.5 \%$ probucol $+0.75 \%$ pantethine (group IX) (Fig. 1). Rabbits were received $150 \mathrm{~g}$ of diet per day and were allowed ad libitum water. Blood samples to determine serum lipids, lipoproteins and apolipoproteins were taken from the ear vein before and after 2, 4, 6 and 9 weeks of treatment. Histopathological examination on arterial atheromatous lesions in cholesterol-fed rabbits in groups II, V, VI and IX (7 animals in each group) was carried out after a 24-week treatment.

Chemicals: Probucol (Lot No.: MM810519, Dow Chemical-Japan, Tokyo) and pantethine (Lot No.: 272, Daiichi Seiyaku Co., Tokyo) were used. Other chemicals used were of reagent grade.

Determination of serum lipids and lipoprotein cholesterols: Serum cholesterol, phospholipids and triglyceride were determined by using enzymatic test kits: Daitest CHO-2 (Daiichi Pure Chemicals Co., Tokyo). Phospholipid B Test Wako (Wako Junyaku Co., Tokyo) and Triglyceride C-lI Test Wako (Wako Junyaku Co., Tokyo) respectively. Serum lipoprotein fractions were prepared by the ultracentrifugation method (18). Serum samples of $150 \mu \mathrm{l}$ each were adjusted to a density of 1.063 by adding $20 \mu$ of $\mathrm{KBr}$ solution $(d=1.491)$ to centrifuge tubes $(0.175$ CN tubes, Hitachikoki, Co., Tokyo). The mixture was centrifuged in an RPL-42T-101 rotor (Hitachikoki, Co., Tokyo) at $128,100 \times \mathrm{g}$ for $16 \mathrm{hr}$ at $16^{\circ} \mathrm{C}$ and the $110 \mu \mathrm{l}$ bottom fraction (HDL) was collected for determination of cholesterol. Cholesterol values in the $V L D L+L D L \quad[(V) L D L]$ fraction were calculated from the difference between total serum cholesterol and HDL cholesterol values.

Determination of serum apolipoproteins: Serum apo A-I and apo B levels were determined by the rocket technique of Laurell (19). One percent agarose gel containing $5 \%$ dextran was prepared in barbital buffer $(\mu=0.05, \mathrm{pH} 8.6$ ) with $5 \%$ anti $\mathrm{A}-1$ serum for apo $A-1$ determination. For apo $B$

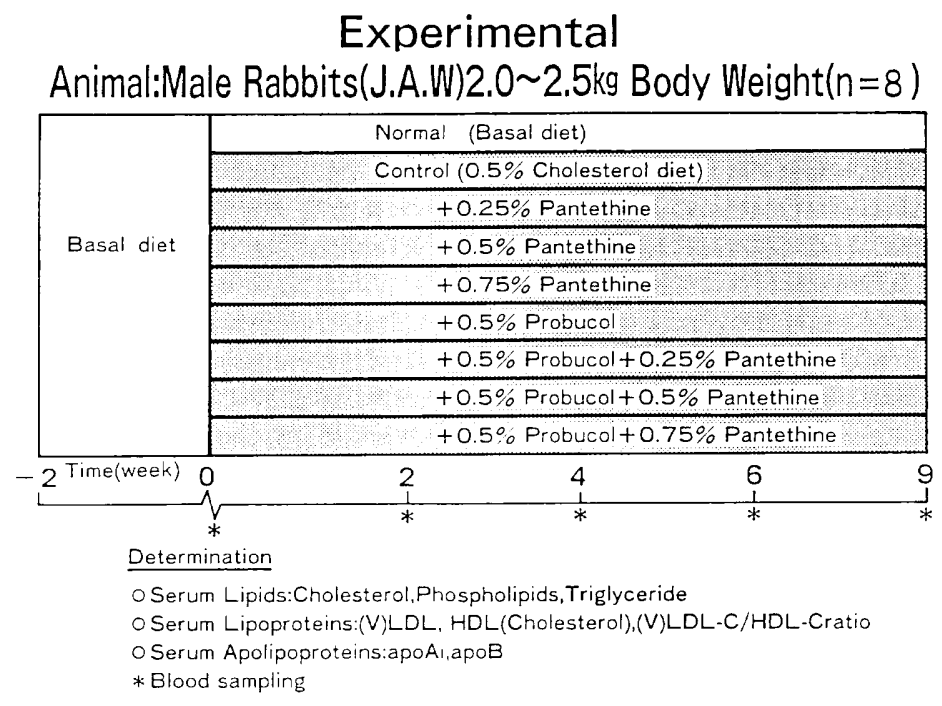

Fig. 1. Experimental schedule 
determination, $0.75 \%$ agarose gel containing $5 \%$ dextran was prepared in barbital buffer $(/=0.025, \mathrm{pH} 8.6)$ with $5 \%$ anti $\mathrm{B}$ serum. The electrophoresis was performed in an electrophoresis apparatus (Marisol Co., Tokyo) and ran for $16 \mathrm{hr}$ at $4^{\circ} \mathrm{C}$ at a field strength of $7 \mathrm{~V} /$ $\mathrm{cm}$ (50V). Samples and the serially diluted serum as reference were applied on the plate. The apolipoprotein concentrations in sera were expressed in $\mathrm{mg} / \mathrm{dl}$.

Pathology: After treatment for 24 weeks, rabbits in groups II, V, VI and IX (7 rabbits in each group) were killed by exsanguination under pentobarbital- $\mathrm{Na}$ anesthesia $(40 \mathrm{mg} /$ kg, i.v.), and the entire aorta and heart were removed. Aorta free of adventitial fat was opened longitudinally and stained with Oilred 0 and photographed to determine the extent of atherosclerotic lesions. The degree of the lesions was graded on a $0-4$ scale according to Duff et al. (20). Tissues (ascending and middle portion of abdominal aorta and heart) were fixed with neutral buffered formalin and then calcified and embedded in paraffin blocks. Cross sections (5-6 $/ \mathrm{m}$ thick) were stained with hematoxyline and eosin $(H-E)$.

Analysis of data: Statistical significance of data was analyzed by Student's $t$-test. Data are presented as the mean \pm S.E.

\section{Results}

Serum lipids and lipoprotein cholesterols: As shown in Table 1. Cholesterol feeding resulted in a great increase in total and (V) LDL cholesterol levels in serum depending on the period. The highest value was observed at the 6th week (103 and 593 fold of normal rabbits, respectively). In contrast, HDL cholesterol level was not significantly affected by cholesterol feeding. Phospholipids levels in serum were also increased (7.5-fold of normal rabbits at the 9th week). Triglyceride level in serum was slightly increased.

In the pantethine-treated groups (groups III. IV and V), total and (V) LDL cholesterol together with phospholipids levels in serum were almost dose-dependently decreased, while HDL cholesterol level was moderately elevated at the 6 and 9th week, and serum triglyceride level was also reduced by only treatment with higher doses.

In the probucol-treated group (group VI), a marked reduction of total and $(V) L D L$ cholesterol levels concomitant with the decrease in HDL cholesterol were observed at all points of measurement during the 9 week period. Phospholipids levels in serum were also decreased but triglyceride level was not affected.

The combined treatment with probucol and pantethine (groups VII, VIII and IX) caused a additive effect in lowering the total and ( $V$ ) LDL cholesterol levels in serum at all the examined periods ( $\sim 9$ weeks). while the reduction of HDL cholesterol level by probucol treatment alone was significantly prevented in a dose-dependent manner by the combination with pantethine. Serum phospholipids levels were also additively reduced by the combinations; however. serum triglyceride level was decreased with no further improvement over the pantethine or probucol treatment alone.

Serum lipids and lipoprotein cholesterol levels in groups II, V, VI and IX at the 18 and 24th week are summarized in Table 2. Almost the same results as seen at the 9 th week were observed.

Serum apolipoproteins: The high cholesterol diet also caused a marked increase in the concentration of serum apo $B$ (about 10-fold that of normal rabbits, Fig. 2); however, the concentration of serum apo A-I was moderately decreased (about 60\% of normal rabbits, Fig. 3) by feeding the cholesterol-supplemented diet for 9 weeks.

Pantethine or probucol treatment reduced serum apo B levels effectively, and these effects were enhanced additively by the combination with both agents (Fig. 2).

Pantethine treatment tended to increase serum apo A-I levels; on the contrary, probucol treatment caused a significant reduction of it (about $60 \%$ ). Combined treatment with these two drugs showed a significant prevention in the reduction of serum apo A-I levels by probucol treatment alone (Fig. 3).

Histopathological observations: The protective effect of pantethine $(0.75 \%$ in diet $)$. probucol $(0.5 \%$ in diet) and their combination $(0.75 \%$ pantethine $+0.5 \%$ probucol in diet) was studied on the 24th week atherosclerotic 


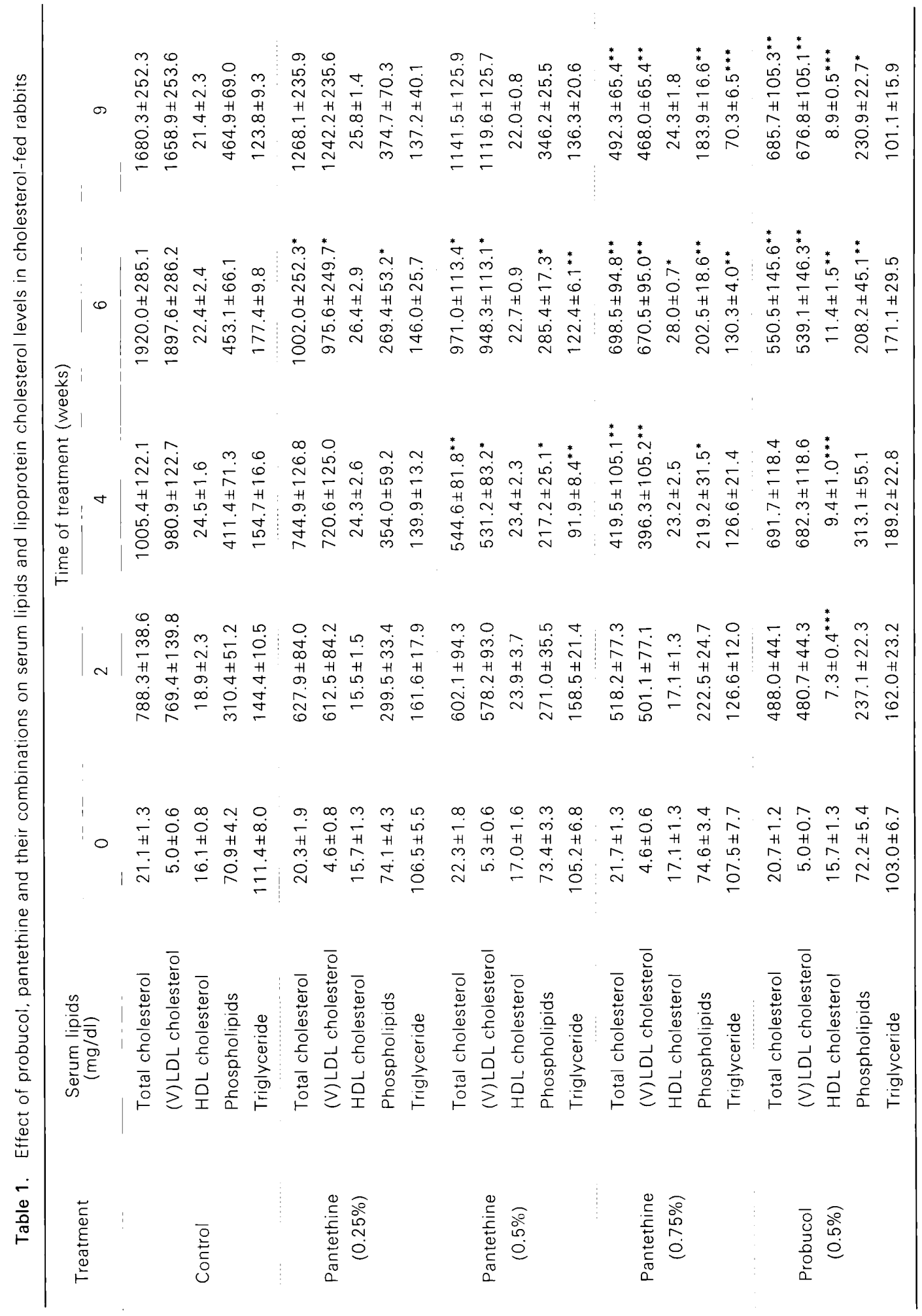




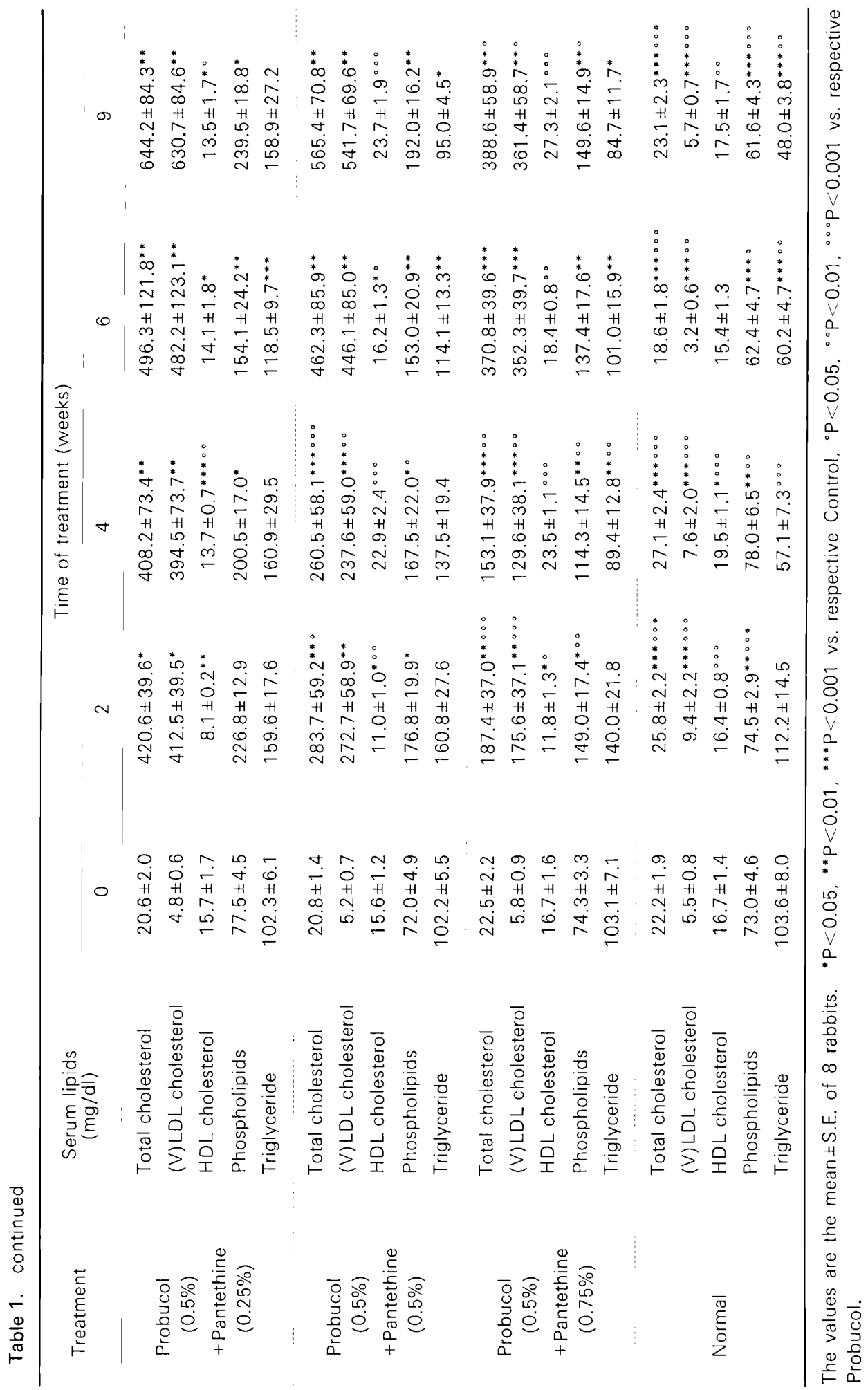




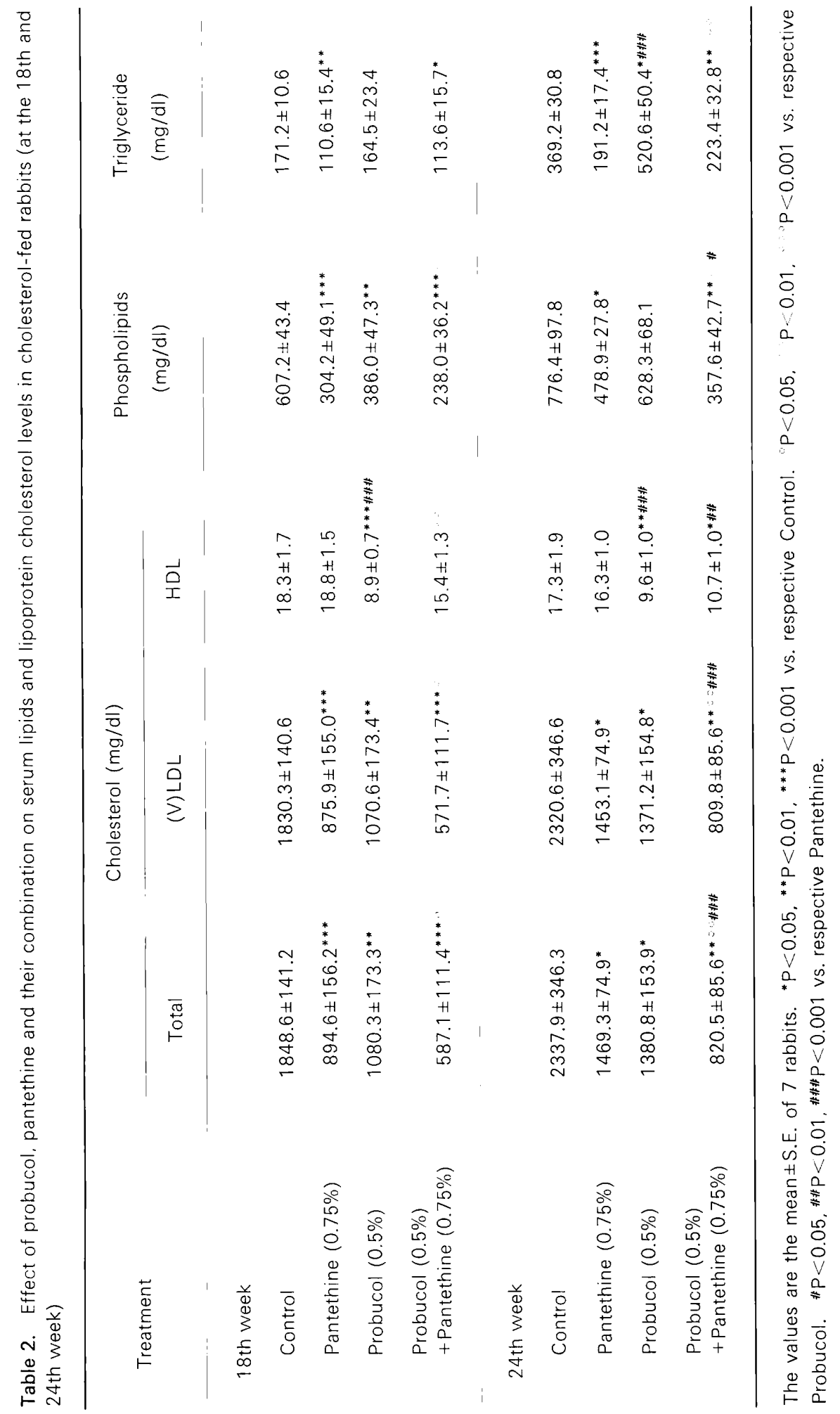


lesions in the aorta and coronary artery of the 4 groups (II, V, VI and IX).

Gross findings: Figure $4 \mathrm{~A}$ shows the atheromatous lesions in the aorta of the control rabbits (group 11 ). The findings are

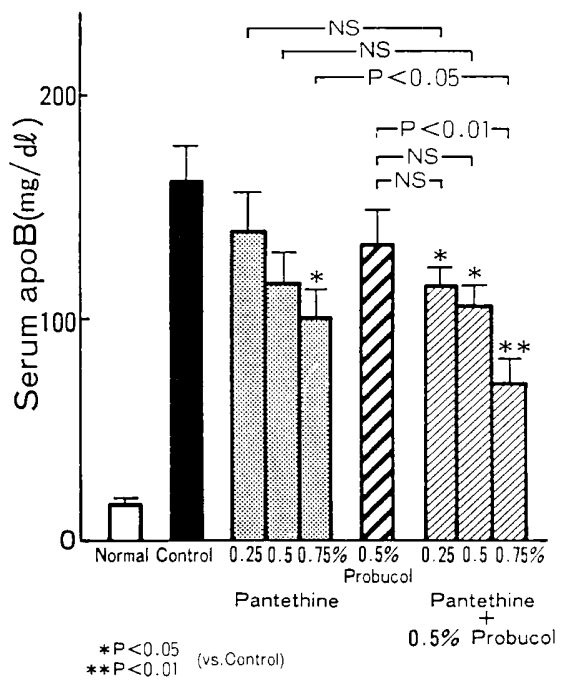

Fig. 2. Effect of probucol, pantethine and their combinations on serum apo $B$ levels in cholesterolfed rabbits (at 9 th week). Each column represents the mean $\pm S$.E. of 8 rabbits. ${ }^{*} \mathrm{P}<0.05,{ }^{*} \mathrm{P}<0.01 \mathrm{vs}$. control.

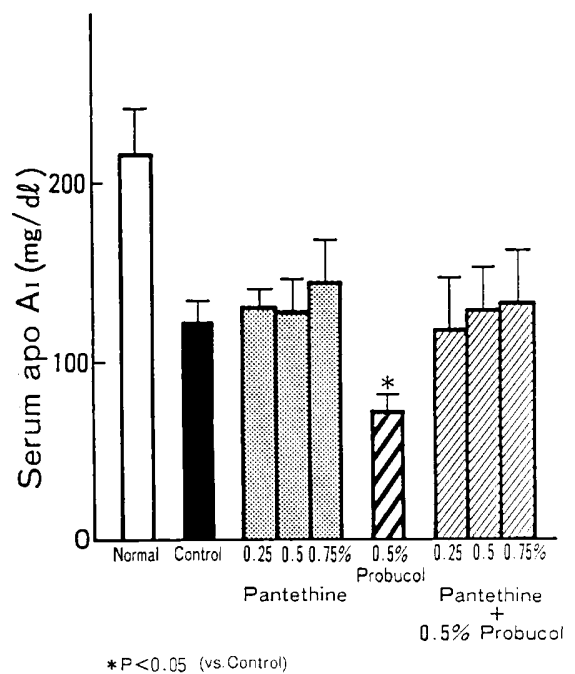

Fig. 3. Effect of probucol, pantethine and their combinations on serum apo A-I levels in cholesterolfed rabbits (at 9 th week). Each column represents the mean $\pm \mathrm{S}$.E. of 8 rabbits. ${ }^{*} P<0.05$ vs. control. characterized as an advanced atherosclerosis, consisting of raised form cell lesions and some plaques covering the entire surface of the artery. Table 3 summarizes the effect of pantethine, probucol and their combination on the extent of aortic lesions after 24 weeks of treatment. Pantethine (group $V$ ) or probucol (group VI) was found to be effective in preventing the progression of atheromatous lesions in the aorta. The combined treatment with pantethine and probucol (group IX) was much more effective than either drug alone (Fig. 4B).

Microscopic findings: Figure 5A shows typical atherosclerotic lesions in the ascending portion of the control rabbit aorta in group II, consisting of intimal thickening with accumulation of foam cells, fibrotic cell proliferation, fragmentation of elastic laminae,

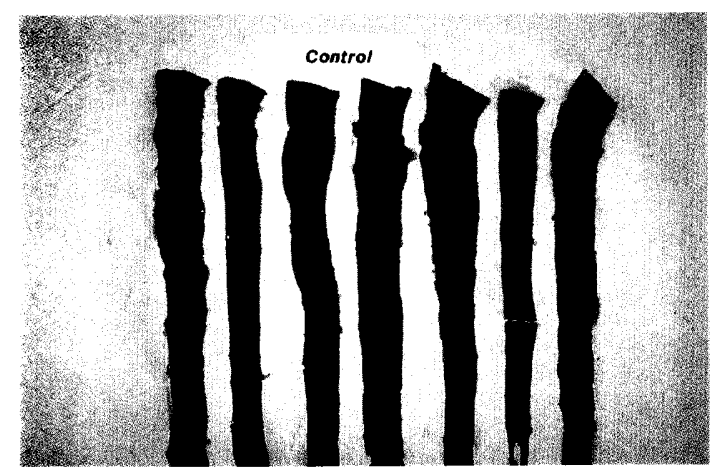

A

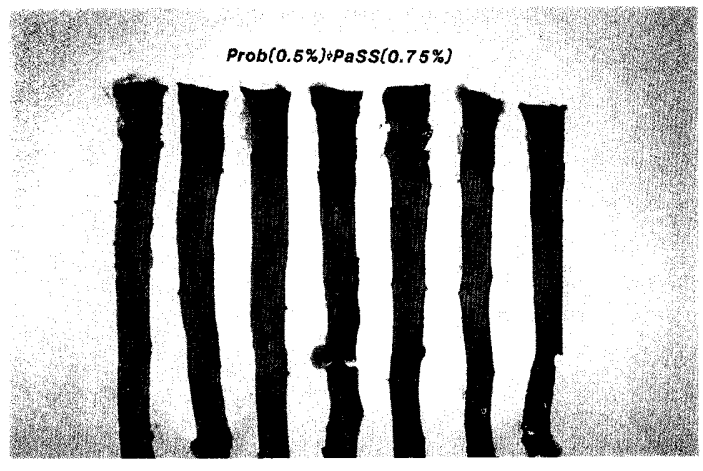

B

Fig. 4. Gross findings in arterial atheromatous lesions (Oil-red 0 stain). (A) Control group (B) Probucol $(0.5 \%)$ plus pantethine $(0.75 \%)$ treated group. 
Table 3. Effect of probucol, pantethine and their combination on the incidence of aortic atheromatous lesions in cholesterol-fed rabbits (gross findings, at the 24th week)

\begin{tabular}{|c|c|c|c|c|c|c|c|}
\hline \multirow{2}{*}{ Treatment } & \multirow{2}{*}{$\begin{array}{l}\text { No. of } \\
\text { rabbits }\end{array}$} & \multicolumn{6}{|c|}{ Degree of aortic lesion } \\
\hline & & 0 & 1 & 2 & 3 & 4 & Mean \pm S.E. \\
\hline Control & 7 & 0 & 0 & 0 & 2 & 5 & $3.7 \pm 0.2$ \\
\hline Pantethine $(0.75 \%)$ & 7 & 0 & 2 & 3 & 2 & 0 & $2.0 \pm 0.3^{* * *}$ \\
\hline Probucol $(0.5 \%)$ & 7 & 1 & 2 & 2 & 2 & 0 & $1.7 \pm 0.4^{* *}$ \\
\hline $\begin{array}{l}\text { Probucol }(0.5 \%) \\
\quad+\text { Pantethine }(0.75 \%)\end{array}$ & 7 & 4 & 2 & 1 & 0 & 0 & $0.6 \pm 0.3^{* * * * * t}$ \\
\hline
\end{tabular}

${ }^{*} \mathrm{P}<0.01$. ${ }^{* *} \mathrm{P}<0.001$ vs. Control. $\mathrm{P}<0.05$ vs. Probucol *\#*P<0.01 vs. Pantethine

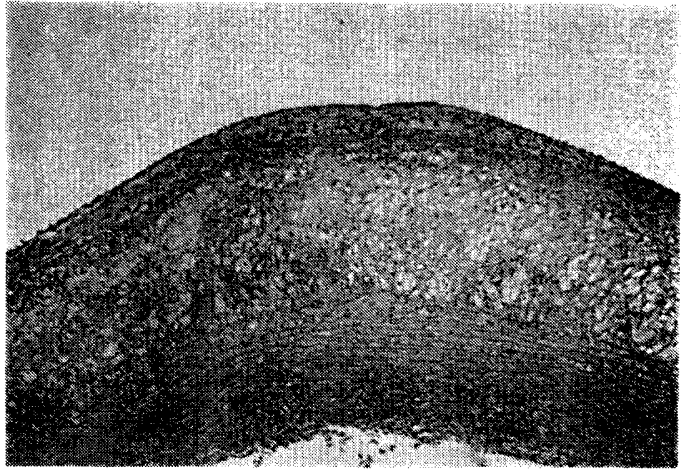

A

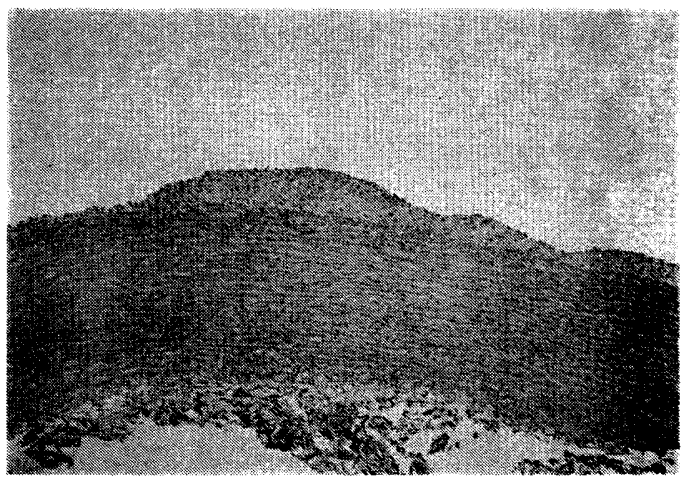

B

Fig. 5. Histological findings in ascending aorta $(H . E$. stain $\times 50)$. (A) Control group (B) Probucol $(0.5 \%)$ plus pantethine $(0.75 \%)$ treated group.

lipid deposition both intra-cellularly and in the extra-cellular space and superficial fibrous capsules in the sections stained with hematoxyline and eosin.

Pantethine or probucol treatment was found to be effective in preventing the

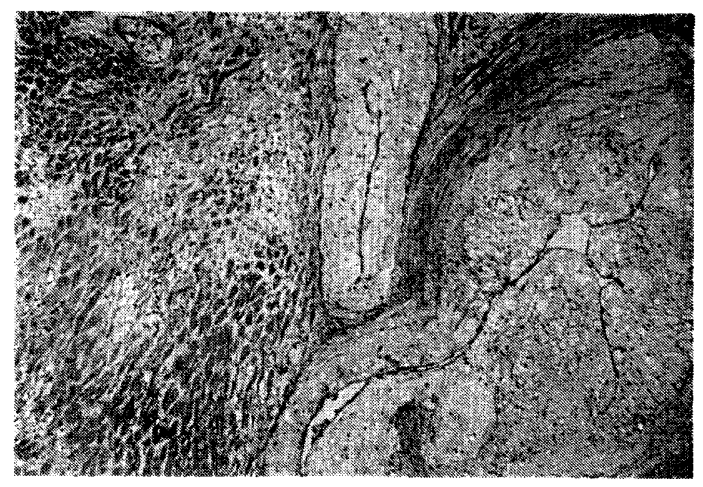

A

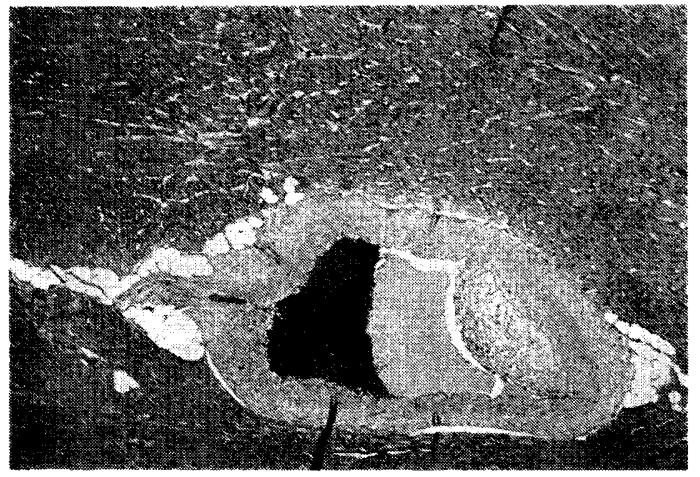

B

Fig. 6. Histological findings in coronary artery (H.E. stain $\times 50)$. (A) Control group (B) Probucol $(0.5 \%)$ plus pantethine $(0.75 \%)$ treated group.

progressin of the atheromatous lesions, i.e., less marked accumulation of the foam cells and inhibition of fibrous cell proliferation in intima and medial cells was observed (photographs not shown). The combined treatment with pantethine and probucol was 


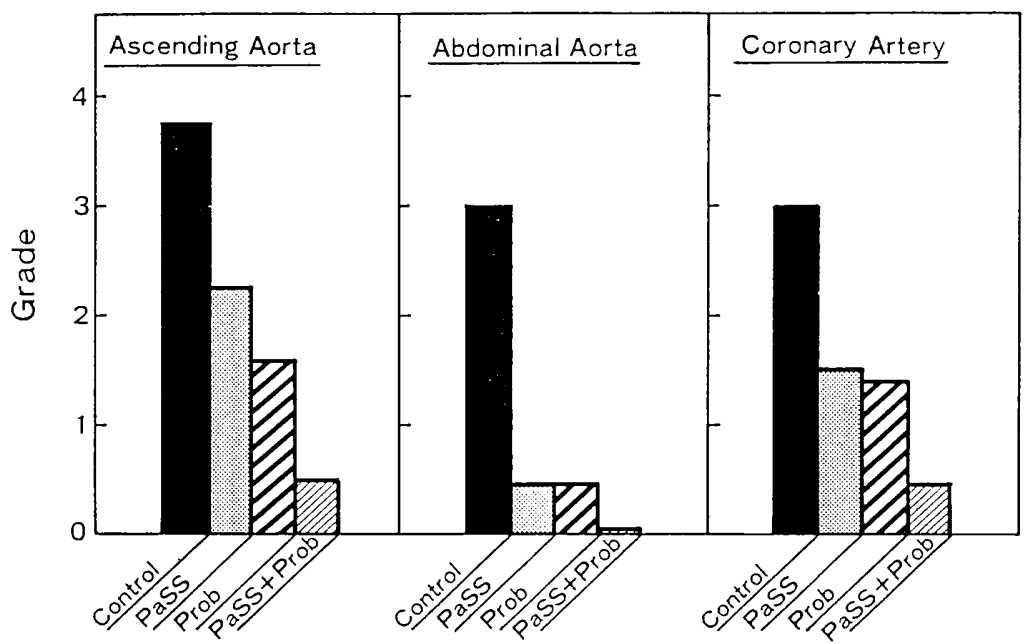

Fig. 7. Effect of probucol, pantethine and their combination on the incidence of atheromatous lesions in aorta and coronary artery in cholesterol-fed rabbits (histological findings, at the 24th week). Each column represents the mean value from 7 rabbits. Prob: probucol (0.5\%), PaSS: pantethine $(0.75 \%)$, PaSS + Prob: pantethine $(0.75 \%)$ plus probucol $(0.5 \%)$.

found to be more effective in preventing the progression of atheromatous lesions in aorta. Only a slight intimal thickening and lipid deposition was observed (Fig. 5B).

Figure $6 \mathrm{~A}$ shows the lesion of coronary artery in the cholesterol-fed control rabbits (group $1 \mathrm{I}$ ). The arterial lumen was greatly narrowed by intimal thickening with accumulation of foam cells and were noted by degeneration of medial smooth muscles and fragmentation of elastic fibers.

Pantethine or probucol treatment resulted in a significant reduction of intimal thickening and a weak foam cell accumulation (Fig. 7, photographs not shown). The combined treatment with pantethine and probucol (group IX) resulted in a highly significant reduction of the degree of the intimal thickening with foam cell accumulation and was found to be highly effective in preventing the progression of atheromatous lesions in the coronary arteries (Figs. 6B and 7).

\section{Discussion}

Associations between low levels of HDL and the risk of developing atherosclerotic disease, specifically of the coronary arteries, have been demonstrated in numerous studies (5-9). However, there were few reports about the fact that lowered HDL cholesterol levels in serum accelerated the progression of atherosclerosis in animal models. In clinical study, a hypolipidemic agent that lowered HDL cholesterol prevented the incidence of coronary arterial diseases (21). More detailed epidemiological studies on a large scale are required to elucidate the relationship between the HDL level and the incidence of atherosclerotic diseases.

Recently, the relationship of the reduction in the incidence of coronary heart disease to the lowering of serum total and LDL cholesterol was clarified in large scale epidemiological studies $(22,23)$.

From the viewpoint of the physiological action of HDL, i.e., removal of stored cholesterol in peripheral tissues, including the aortic wall, to the liver, it might be more effective if the lowering activity of probucol on HDL cholesterol and serum apo A-I levels could be compensated by some means. The present study was carried out with two major objectives (a) to clarify whether the reduction of HDL cholesterol and serum apo A-I levels by probucol treatment could be compensated by the combination with other agents such as HDL-elevating agents like "pantethine", an intermediate precursor of coenzyme A. (b) to clarify whether the improvement of serum 
lipoprotein disorders could have a favorable influence on arterial atheromatous lesions.

In the present study with hyperlipidemic rabbits, probucol treatment $(0.5 \%$ in diet $)$ resulted in a marked reduction in serum total and (V)LDL cholesterol levels. A significant reduction of HDL cholesterol and serum apo A-I levels was also observed in probucol treated rabbits. On the other hand, pantethine treatment $(0.25 \%-0.75 \%$ in diet) caused a dose-dependent reduction in serum total and (V) LDL cholesterol levels. HDL cholesterol and serum apo A-I levels were moderately elevated in this experiment with little difference from that of the previous report (18). The combination of probucol with pantethine resulted in a synergistic effect in lowering the serum total and (V)LDL cholesterol levels, while their combinations exerted a significant dose-dependent prevention for the decrease of HDL cholesterol and serum apo A-I levels by probucol treatment alone (Table 1 and Fig. 3).

In clinical studies, several combination therapies with different types of hypolipidemic agents have been reported. Cholestylamine and probucol combination therapy in familial hypercholesterolemia can result in a greater reduction in serum cholesterol than with either drug alone, but failed to compensate for the reduction of HDL cholesterol by probucol (24). Mizuno et al. (25) reported that a combined administration of probucol and pantethine in heterozygous familial hypercholesterolemia was effective in preventing the HDL cholesterol reduction by probucol in addition to synergistic decrease in serum cholesterol.

It has been reported that probucol reduced HDL cholesterol (apo A-I) by inhibiting the biosynthesis of apo $A-1$ in the organs from the kinetic study of labelled HDL (26), although the detailed mechanism of probucol with regards to the decrease in HDL has not been clear yet.

On the other hand, pantethine increased $\mathrm{HDL}$ cholesterol through the stimulation of the VLDL-HDL pathway, i.e., the heparinreleasable lipoprotein lipase activity in the epididymal adipose tissue as well as the lecithin: cholesterol acyltransferase (ACAT) activity in serum was markedly increased by treatment with pantethine (27). Pantethine treatment also resulted in an acceleration of the biosynthesis of apo $A-1$ in the perfusion system of isolated rabbit livers (28). Therefore, it has been speculated that these opposite actions of the combined drugs on HDL cholesterol and apo A-I might contribute to their compensatory actions.

In this study, the severity of atheromatous lesions in aorta and coronary arteries in cholesterol-fed rabbits was effectively prevented by probucol or pantethine (Fig. 7). These findings are consistent with the results of Krichevsky et al. (29) in the probucol study and with the results of Carrara et al. (30) in the pantethine study.

The protective effect of pantethine may be possibly due to the increased HDL cholesterol levels and the marked reduction in the serum levels of atherogenic (V)LDL. Pantethine also has other actions, i.e., increasing the arterial cholesterol ester hydrolase activities (31). It may be related to the protective effect of pantethine on atheromatous lesions in the artery.

Protective effect of probucol may be related mainly to the marked reduction of atherogenic (V)LDL in the serum. The reduction of HDL cholesterol may be less important than the reduction of $(V) L D L$ cholesterol for the progression of atheromatous lesions in this animal models, because (V) LDL cholesterol level in the cholesterolfed rabbit was extremly high, and HDL cholesterol level was extremly low. There is another possible action for the protective effect of probucol in the progression of atherosclerosis: it has the advantage of inhibiting the oxidation of LDL in cultured endothelial cells and macrophages (32). Combined treatment with probucol and pantethine excerted a synergistic effect in preventing the progression of arterial atheromatous lesions both in aorta and in coronary arteries (Fig. 7). This marked protective effect in their combination therapy would be related to the cummulative actions of these two agents mentioned above.

In conclusion, the combined treatment with probucol and pantethine has a favorable influence on the metabolism of serum lipoproteins and has a protective effect in the 
progression of atheromatous lesions in the aorta and coronary arteries in cholesterol-fed rabbits.

\section{References}

1 Keys, A., Aravanis, C. and Blackburn, H.: Probability of middle-aged men developing coronary heart disease in five years. Circulation 45, 815-828 (1972)

2 Pooling Project Research Group: Relationship of blood pressure, serum cholesterol, smoking habit, relative weight, and ECG abnormalities to incidence of major coronary events: Final report of the pooling project. J. Chronic Dis. 31, 201306 (1978)

3 Kannel, W.B., Castelli, W.P. and Gordon, T.: Cholesterol in the prediction of atherosclerotic disease. New prospectives on the framingham study. Ann. Intern. Med. 90, 85 (1979)

4 Levy, R.l. and Feinlieb, M.: Risk factors for coronary artery disease and their management. In Heart Disease. Edited by Braunwald. E., p. 1246-1278, W.B. Saunders Co., Philadelphia (1980)

5 Rhoadas, G.G., Gulbrandsen, C.L. and Kagan, A.: Serum lipoproteins and coronary heart disease in a population study of Hawaii Japanese men. N. Engl. J. Med. 294. 293-298 (1976)

6 Miller, G.J. and Miller, N.E.: Plasma high density lipoprotein concentration and development of ischemic heart disease. Lancet 1, 16-19 (1975)

7 Gordon, T.W., Castelli, W.P., Hjortland, M.C., Kannel, W.B. and Dawber, T.R.: High density lipoprotein as a protective factor against coronary heart disease. Am. J. Med. 62, 707-714 (1977)

8 Miller, N.E., Förde, O.H., Thelle, D.S. and Mjös, O.D.: High density lipoprotein and coronary heart disease: A prospective case-control study. Lancet 2, 965-968 (1977)

9 William, P.C., Joseph, T.D., Stephen, B.H., Abraham, K. and William, J.Z.: HDL cholesterol and other lipids in coronary heart disease. The cooperative lipoprotein phenotyping study. Circulation 55, 767-773 (1977)

10 Murphy, B.F.: Probucol in treatment of hyperlipidemia. JAMA 238, 2537-2538 (1977)

11 Nash, D.T.: Safety and efficiency of probucol during one year of administration. J. Clin. Pharmacol. 14, 470-475 (1974)

12 Barnhart, J.W., Rytter, D.J. and Morello, J.A.: An overview of the biochemical pharmacology of probucol. Lipids 12, 29-33 (1977)

13 LeLorier, J., DuBreuil-Quindoz, S., LussierCacan, S., Huang, Y.S. and Davingnon, J.: Diet and probucol in lowering cholesterol concen- trations. Arch. Int. Med. 137, 116-121 (1977)

14 Barnhart, J.W., Sefranka, J.A. and Mclntosh, D.D.: Hypocholesterolemic effect of $4.4^{\prime}$-(isopropyl-idenedithio) - bis - (2,6-di-t-butylphenol) (probucol). Am. J. Clin. Nutr. 23, 1229-1233 (1970)

15 Tomikawa, M., Nakayasu, T., Tawara, K. and Abiko, Y.: Effect of probucol on serum lipoprotein levels in normal and dyslipoproteinemic mice. Atherosclerosis 40,101-113 (1981)

16 Miettinen, T.A., Huttunen, J.K., Ehnholm, C., Kumlins, T., Mattila, S. and Naukkarinen, V.: Effect of long-term antihypertensive and hypolipidemic treatment on high density lipoprotein cholesterol and apoproteins $A-1$ and A-11. Atherosclerosis 36, 249-259 (1980)

17 Mondasini, R., Keller, M. and Riesen, W.F.: Effect of probucol and diet on serum lipids and lipoprotein fractions in primary hypercholesterolemia. In Diet and Drug in Atheroscierosis, Edited by Noseda, C., Lewis, B. and Paoletti, R., p. 181-187, Raven press, New York (1980)

18 Tomikawa, M., Nakayasu, T., Tawara, K., Kameda, K. and Abiko, Y.: Effect of pantethine on lipoprotein profiles and HDL subfractions in experimentally hypercholesterolemic rabbits. Atherosclerosis 41, 267-277 (1982)

19 Laurell, C.B.: Electroimmunoassay. Scand. J. Clin. Lab. Invest. 29, Supp. 124, 21-37 (1972)

20 Duff, G.L. and McMillian, G.C.: The effect of alloxan diabetes on experimental atherosclerosis in the rabbit. J. Exp. Med. 89, 611-630 (1949)

21 Miettinen, T.A., Huttunen, J.K., Kumlin, T., Naukkarinen, V., Mattila, S. and Ehnholm, C.: Lowered HDL-cholesterol and incidence of ischemic heart disease. Lancet 2, 478 (1981)

22 Lipid Research Clinics Program: The lipid research clinics coronary primary prevention trial results. I. Reduction in incidence of coronary heart disease. JAMA 251, 351-364 (1984)

23 Lipid Research Clinics Program: The lipid research clinics coronary primary prevention trial results. 11. Relationship of reduction in incidence of coronary heart disease to cholesterol lowering. JAMA 251, 365-374 (1984)

24 Jackson, J.M. and Lee, H.A.: The effect of probucol and cholestyramine combination therapy in severe familial hypercholesterolemia. Atherosclerosis 51, 189-197 (1984)

25 Mizuno, G., Hara, Y., Shimizu, T., Tochihara, T., Izumida, H., Kishi, H., Fujioka, T. and Yasugi, T.: Preventive effects of pantethine to reduction of HDL cholesterol caused by probucol administration. J. Japan. Atheroscler. Soc. 12, 191-197 (1984) 
26 Nestel, P.J. and Billington, T.: Effects of probucol on low density lipoprotein removal and high density lipoprotein synthesis. Atherosclerosis 38, 203-209 (1981)

27 Tomikawa, M., Tawara, K., Nakayasu, T., Kameda, K. and Abiko, Y.: Improvement of lipoprotein metabolism in experimental hyperLDL rabbits by pantethine. In Abstracts of 12 th International Congress of Gerontology, Hamburg (1981)

28 Tomikawa, M., Nakayasu, T., Wakasugi, J. and Abiko, Y.: Effect of pantethine on HDL metabolism in rabbits. In Proceedings of 5 th Symposium on Pantethine, p. 181-192. Daiichi Seiyaku Press, Tokyo (1981)

29 Kritchevsky, D., Kim, H.K. and Tepper, A.T.: Influence of 4,4-(isopropylidene-dithio)-bis(2.6-di-t-butylphenol) ( $\mathrm{DH}-581)$ on experi- mental atherosclerosis in rabbits. P.S.E.B.M. 136, 1216-1221 (1970)

30 Carrara, P., Matturi, L., Ga!bussera, M., Lovati, M.R., Franceschini, G. and Sirtori, C.R.: Pantethine reduces plasma cholesterol and the severity of arterial lesions in experimental hypercholesterolemic rabbits. Atherosclerosis 53, 255-264 (1984)

31 Shinomiya, M., Matsuoka, N., Shirai, K., Morisaki, N., Sasaki, N., Murano, S., Saito, Y. and Kumagai, A.: Effect of pantethine on cholesterol ester metabolism in rat arterial wall. Atherosclerosis 36, 75-80 (1980)

32 Steinberg, D.: Endothelia! cell (EC)-modification of low density lipoprotein (LDL): The essential role of phospholipase activity. In Abstracts of 7 th International Symposium on Atherosclerosis, Melbourne (1985) 